\title{
An Asymptotic Analysis of Generative, Discriminative, and Pseudolikelihood Estimators
}

Percy Liang

PLIANG@CS.BERKELEY.EDU

Computer Science Division, University of California, Berkeley, CA, USA

Michael I. Jordan

JORDAN@CS.BERKELEY.EDU

Computer Science Division and Department of Statistics, University of California, Berkeley, CA, USA

\begin{abstract}
Statistical and computational concerns have motivated parameter estimators based on various forms of likelihood, e.g., joint, conditional, and pseudolikelihood. In this paper, we present a unified framework for studying these estimators, which allows us to compare their relative (statistical) efficiencies. Our asymptotic analysis suggests that modeling more of the data tends to reduce variance, but at the cost of being more sensitive to model misspecification. We present experiments validating our analysis.
\end{abstract}

\section{Introduction}

Probabilistic models play a prominent role in domains such as natural language processing, bioinformatics, and computer vision, where they provide methods for jointly reasoning about many interdependent variables. For prediction tasks, one generally models a conditional distribution over outputs given an input. There can be reasons, however, for pursuing alternatives to conditional modeling. First, we might be able to leverage additional statistical strength present in the input by using generative methods rather than discriminative ones. Second, the exact inference required for a full conditional likelihood could be intractable; in this case, one might turn to computationally more efficient alternatives such as pseudolikelihood (Besag, 1975).

The generative-discriminative distinction has received much attention in machine learning. The standing intuition is that while discriminative methods achieve lower asymptotic error, generative methods might be

Appearing in Proceedings of the $25^{\text {th }}$ International Conference on Machine Learning, Helsinki, Finland, 2008. Copyright 2008 by the author(s)/owner(s). better when training data are limited. This intuition is supported by the theoretical comparison of Naive Bayes and logistic regression ( $\mathrm{Ng} \&$ Jordan, 2002) and the recent empirical success of hybrid methods (McCallum et al., 2006; Lasserre et al., 2006).

Computational concerns have also spurred the development of alternatives to the full likelihood; these methods can be seen as optimizing an alternate objective or performing approximate inference during optimization. Examples include pseudolikelihood (Besag, 1975), composite likelihood (Lindsay, 1988), tree-reweighted belief propagation (Wainwright et al., 2003), piecewise training (Sutton \& McCallum, 2005), agreement-based learning (Liang et al., 2008), and many others (Varin, 2008).

We can think of all these schemes as simply different estimators operating in a single model family. In this work, we analyze the statistical properties of a class of convex composite likelihood estimators for exponential families, which contains the generative, discriminative, and pseudolikelihood estimators as special cases.

The main focus of our analysis is on prediction error. Standard tools from learning theory based on uniform convergence typically only provide upper bounds on this quantity. Moreover, they generally express estimation error in terms of the overall complexity of the model family. ${ }^{1}$ In our case, since all estimators operate in the same model family, these tools are inadequate for comparing different estimators.

Instead, we turn to asymptotic analysis, a mainstay of theoretical statistics. There is much relevant statistical work on the estimators that we treat; note in particular that Lindsay (1988) used asymptotic arguments to show that composite likelihoods are generally

\footnotetext{
${ }^{1}$ There are more advanced techniques such as local Rademacher complexities, which focus on the relevant regions of the model family, but these typically only apply to empirical risk minimization.
} 
less efficient than the joint likelihood. The majority of these results are, however, focused on parameter estimation. In the current paper, our focus is on prediction, and we also consider model misspecification.

We draw two main conclusions from our analysis: First, when the model is well-specified, conditioning on fewer variables increases statistical efficiency; this to some extent accounts for the better generalization enjoyed by generative estimators and the worse performance of pseudolikelihood estimators. Second, model misspecification can severely increase both the approximation and estimation errors of generative estimators. We confirm our theoretical results by comparing our three estimators on a toy example to verify the asymptotics and on a Markov model for part-of-speech tagging.

\section{Exponential Family Estimators}

In structured prediction tasks, we are interested in learning a mapping from an input space $\mathcal{X}$ to an output space $\mathcal{Y}$. Probabilistic modeling is a common platform for solving such tasks, allowing for the natural handling of missing data and the incorporation of latent variables.

In this paper, we focus on regular exponential families, which define distributions over an outcome space $\mathcal{Z}$ as follows:

$$
p_{\theta}(z) \stackrel{\text { def }}{=} \exp \left\{\phi(z)^{\top} \theta-A(\theta)\right\} \text { for } z \in \mathcal{Z},
$$

where $\phi(z) \in \mathbb{R}^{d}$ is a vector of sufficient statistics (features), $\theta \in \mathbb{R}^{d}$ is a vector of parameters, and $A(\theta) \stackrel{\text { def }}{=} \log \int e^{\phi(z)^{\top} \theta} \nu(d z)$ is the log-partition function. In our case, the outcomes are input-output pairs: $z=(x, y)$ and $\mathcal{Z}=\mathcal{X} \times \mathcal{Y}$.

Exponential families include a wide range of popular models used in machine learning. For example, for a conditional random field (CRF) (Lafferty et al., 2001) defined on a graph $G=(V, E)$, we have an output variable for each node $\left(y=\left\{y_{i}\right\}_{i \in V}\right)$, and the features are $\phi(x, y)=\sum_{i \in V} \phi_{\text {node }}\left(y_{i}, \mathbf{x}, i\right)+\sum_{(i, j) \in E} \phi_{\text {edge }}\left(y_{i}, y_{j}\right)$.

From the density $p_{\theta}(z)$, we can compute event probabilities as follows:

$$
p_{\theta}(z \in s)=\exp \{A(\theta ; s)-A(\theta)\},
$$

where $A(\theta ; s)=\log \int e^{\phi(z)^{\top} \theta} \mathbb{1}[z \in s] \nu(d z)$ is a conditional log-partition function.

\subsection{Composite Likelihood Estimators}

In this paper, we consider a class of composite likelihood estimators (Lindsay, 1988), which is incidentally equivalent to the multi-conditional learning framework of McCallum et al. (2006). A composite likelihood consists of a weighted sum of component likelihoods, each of which is the probability of one subset of variables conditioned on another. In this work, we only consider the case where the first set is all the variables.

We adopt the following more fundamental way of specifying the components: Each component $r$ is defined by a partitioning of the outcome space $\mathcal{Z}$. We represent a partitioning by an associated equivalence function that maps each $z \in \mathcal{Z}$ to its partition:

Definition 1 (Equivalence function). An equivalence function $r$ is a measurable map from $\mathcal{Z}$ to measurable subsets of $\mathcal{Z}$ such that for each $z \in \mathcal{Z}$ and $z^{\prime} \in r(z)$, $r(z)=r\left(z^{\prime}\right)$.

The component likelihood associated with $r$ takes the following form:

$$
p_{\theta}(z \mid z \in r(z))=\exp \left\{\phi(z)^{\top} \theta-A(\theta ; r(z))\right\} .
$$

By maximizing this quantity, we are intuitively taking probability mass away from some neighborhood $r(z)$ of $z$ and putting it on $z$.

Without loss of generality, assume the component weights sum to 1 , so we can think of taking an expectation over a random component $R$ drawn from some fixed distribution $P_{\mathrm{r}}$. We then define the criterion function:

$$
m_{\theta}(z) \stackrel{\text { def }}{=} \mathbb{E}_{R \sim P_{\mathrm{r}}} \log p_{\theta}(z \mid z \in R(z)) .
$$

Given data points $Z^{(1)}, \ldots, Z^{(n)}$ drawn i.i.d. from some true distribution $p^{*}$ (not necessarily in the exponential family), the maximum composite likelihood estimator is defined by averaging the criterion function over these data points:

$$
\hat{\theta}=\underset{\theta}{\operatorname{argmax}} \hat{\mathbb{E}} m_{\theta}(Z),
$$

where $\hat{\mathbb{E}} m_{\theta}(Z)=\frac{1}{n} \sum_{i=1}^{n} m_{\theta}\left(Z^{(i)}\right)$.

We can now place the three estimators of interest in our framework:

Generative: We have one component $r_{\mathrm{g}}(x, y)=\mathcal{X} \times$ $\mathcal{Y}$, which has one partition - the whole outcome space.

Fully discriminative: We have one component $r_{\mathrm{d}}(x, y)=x \times \mathcal{Y}$. The outcomes in each partition have the same value of $x$, but different $y$.

Pseudolikelihood discriminative: Assume $y=$ $\left\{y_{i}\right\}_{i \in V}$. For each $i \in V$, we have a component $r_{i}(x, y)=\left\{\left(x^{\prime}, y^{\prime}\right): x^{\prime}=x, y^{\prime} \in \mathcal{Y}, y_{j}^{\prime}=y_{j}\right.$ for $\left.j \neq i\right\}$. $P_{\mathrm{r}}$ is uniform over these components. 


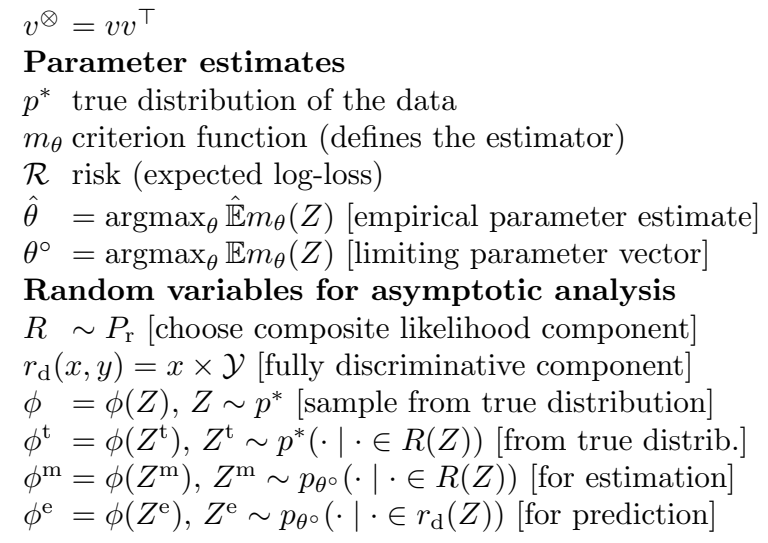

Table 1. Notation used in the paper.

\subsection{Prediction and Evaluation}

Given a parameter estimate $\hat{\theta}$, we make predictions based on $p_{\hat{\theta}}(y \mid x)$. In this paper, we evaluate our model according to log-loss; the risk is the expected log-loss:

$$
\mathcal{R}(\theta)=\mathbb{E}_{(X, Y) \sim p^{*}}\left[-\log p_{\theta}(Y \mid X)\right] .
$$

The quality of an estimator is determined by the gap between the risk of the estimate $\mathcal{R}(\hat{\theta})$ and the Bayes risk $\mathcal{R}^{*}=H(Y \mid X)$. It will be useful to relate these two via the risk of $\theta^{\circ}=\operatorname{argmax}_{\theta} \mathbb{E}_{Z \sim p^{*}} m_{\theta}(Z)$, which leads to the following standard decomposition:

$$
\underbrace{\mathcal{R}(\hat{\theta})-\mathcal{R}^{*}}_{\text {total error }}=\underbrace{\left(\mathcal{R}(\hat{\theta})-\mathcal{R}\left(\theta^{\circ}\right)\right)}_{\text {estimation error }}+\underbrace{\left(\mathcal{R}\left(\theta^{\circ}\right)-\mathcal{R}^{*}\right)}_{\text {approx. error }} .
$$

The estimation error is due to having only finite data; the approximation error is due to the intrinsic suboptimality of the estimator. ${ }^{2}$

\section{Asymptotic Analysis}

We first compute the asymptotic estimation errors of composite likelihood estimators in general (Sections 3.1 and 3.2). Then we use these results to compare the estimators of interest (Sections 3.3 and 3.4).

In this paper, we assume that our exponential family is identifiable. ${ }^{3}$ Also assume that our estimators converge $\left(\hat{\theta} \stackrel{P}{\longrightarrow} \theta^{\circ}\right)$ and are consistent when the model is

\footnotetext{
${ }^{2}$ Note that $\theta^{\circ}$ is not necessarily the minimum risk parameter vector in the model family.

${ }^{3}$ In the non-identifiable case, the analysis becomes more cluttered, but the results are essentially the same, since predictions depend on only the distributions induced by the parameters. See the longer version of this paper for an in-depth discussion.
}

well-specified (if $p^{*}=p_{\theta^{*}}$, then $\theta^{\circ}=\theta^{*}$ ). Note, however, that in general we do not assume that our model is well-specified.

Our asymptotic analysis is driven by Taylor expansions, so we need to compute a few derivatives. The derivatives of the log-partition function are moments of the sufficient statistics (a standard result, see Wainwright and Jordan (2003)):

$$
\begin{aligned}
& \dot{A}(\theta ; s)=\mathbb{E}_{Z \sim p_{\theta}(\cdot \mid \cdot \in s)}(\phi(Z)) \\
& \ddot{A}(\theta ; s)=\operatorname{var}_{Z \sim p_{\theta}(\cdot \mid \cdot \in s)}(\phi(Z)) .
\end{aligned}
$$

From these moments, we can obtain the derivatives of $m_{\theta^{\circ}}$ and $\mathcal{R}$ (to simplify notation, we express these in terms of random variables whose distributions are defined in Table 1):

$$
\begin{aligned}
\dot{m}_{\theta^{\circ}} & =\phi-\mathbb{E}\left(\phi^{\mathrm{m}} \mid Z\right) \\
\ddot{m}_{\theta^{\circ}} & =-\mathbb{E}\left[\operatorname{var}\left(\phi^{\mathrm{m}} \mid R(Z)\right) \mid Z\right] \\
\dot{\mathcal{R}}\left(\theta^{\circ}\right) & =\mathbb{E}\left(\phi^{\mathrm{e}}-\phi\right) \\
\ddot{\mathcal{R}}\left(\theta^{\circ}\right) & =\mathbb{E} \operatorname{var}\left(\phi^{\mathrm{e}} \mid Z\right) .
\end{aligned}
$$

\subsection{Asymptotics of the Parameters}

We first analyze how fast $\hat{\theta}$ converges to $\theta^{\circ}$ by computing the asymptotic distribution of $\hat{\theta}-\theta^{\circ}$. In Section 3.2 we use this result to get the asymptotic distribution of the estimation error $\mathcal{R}(\hat{\theta})-\mathcal{R}\left(\theta^{\circ}\right)$.

The following standard lemma will prove to be very useful in our analysis:

Lemma 1. For random vectors $X, Y, Z$, we have $\operatorname{var}(X \mid Z)=\mathbb{E}[\operatorname{var}(X \mid Y, Z) \mid Z]+\operatorname{var}[\mathbb{E}(X \mid Y, Z) \mid$ $Z$ ].

The important implication of this lemma is that conditioning on another variable $Y$ reduces the variance of $X$. This lemma already hints at how conditioning on more variables can lead to poorer estimators: conditioning reduces the variance of the data, which can make it harder to learn about the parameters.

The following theorem gives us the asymptotic variance of a general composite likelihood estimator:

Theorem 1 (Asymptotic distribution of the parameters). Assume $\hat{\theta} \stackrel{P}{\longrightarrow} \theta^{\circ}$. Then

$$
\sqrt{n}\left(\hat{\theta}-\theta^{\circ}\right) \rightarrow \mathcal{N}(0, \Sigma) .
$$

The asymptotic variance is

$$
\Sigma=\Gamma^{-1}+\Gamma^{-1}\left(C_{\mathrm{c}}+C_{\mathrm{m}}\right) \Gamma^{-1},
$$

where $\Gamma=\mathbb{E} \operatorname{var}\left(\phi^{\mathrm{m}} \mid R(Z)\right)$ is the sensitivity, $C_{\mathrm{c}}=$ $\mathbb{E} \operatorname{var}\left[\mathbb{E}\left(\phi^{\mathrm{m}} \mid R(Z)\right) \mid Z\right]$ is the component correction, and $C_{\mathrm{m}}=\mathbb{E}\left[\operatorname{var}\left(\phi^{\mathrm{t}} \mid Z\right)-\operatorname{var}\left(\phi^{\mathrm{m}} \mid Z\right)\right]+\mathbb{E}\left[\mathbb{E}\left(\phi^{\mathrm{t}} \mid\right.\right.$ $\left.Z)-\mathbb{E}\left(\phi^{\mathrm{m}} \mid Z\right)\right]^{\otimes}$ is the misspecification correction. 
Proof. The standard asymptotic normality result for M-estimators (Theorem 5.21 of van der Vaart (1998)), which includes composite likelihood estimators, gives us the asymptotic variance:

$$
\Sigma=\left(\mathbb{E} \ddot{m}_{\theta^{\circ}}\right)^{-1}\left(\mathbb{E} \dot{m}_{\theta^{\circ}}^{\otimes}\right)\left(\mathbb{E} \ddot{m}_{\theta^{\circ}}\right)^{-1} .
$$

The remainder of the proof simply re-expresses $\Sigma$ in terms of more interpretable quantities. Algebraic manipulation of (10) yields:

$$
\mathbb{E} \dot{m}_{\theta^{\circ}}^{\otimes}=\mathbb{E}\left[\left(\phi-\mathbb{E}\left(\phi^{\mathrm{t}} \mid Z\right)\right)+\left(\mathbb{E}\left(\phi^{\mathrm{t}} \mid Z\right)-\mathbb{E}\left(\phi^{\mathrm{m}} \mid Z\right)\right)\right]^{\otimes} .
$$

Note that cross terms cancel conditioned on $Z$ and that $\mathbb{E}\left[\phi-\mathbb{E}\left(\phi^{\mathrm{t}} \mid Z\right)\right]^{\otimes}=\mathbb{E}\left[\phi^{\mathrm{t}}-\mathbb{E}\left(\phi^{\mathrm{t}} \mid Z\right)\right]^{\otimes}$, so

$$
\mathbb{E} \dot{m}_{\theta^{\circ}}^{\otimes}=C_{\mathrm{m}}+\mathbb{E} \operatorname{var}\left(\phi^{\mathrm{m}} \mid Z\right) .
$$

We then apply Lemma 1 to decompose the second term of the right-hand side:

$$
\begin{aligned}
& \mathbb{E} \operatorname{var}\left(\phi^{\mathrm{m}} \mid Z\right)= \\
& \quad \mathbb{E} \operatorname{var}\left(\phi^{\mathrm{m}} \mid R(Z)\right)+\mathbb{E} \operatorname{var}\left[\mathbb{E}\left(\phi^{\mathrm{m}} \mid R(Z)\right) \mid Z\right]
\end{aligned}
$$

Substitute (18) into (17) to get an expression for $\mathbb{E} \dot{m}_{\theta^{\circ}}^{\otimes}$ (11) already provides one for $\mathbb{E} \ddot{m}_{\theta^{\circ}}$. Substitute these two expressions into (16) and simplify to get (15).

The decomposition in (15) allows us to make several qualitative judgments. First, the sensitivity $\Gamma=$ $\mathbb{E} \operatorname{var}\left(\phi^{\mathrm{m}} \mid R(Z)\right)$ is the expected amount of variation in the features given $Z$ and $R$ (equivalently, given $R(Z)$ ). The larger the sensitivity, the more the data can tell us about the parameters, and thus the lower the asymptotic variance will be.

The component correction $C_{\mathrm{c}}$ intuitively measures how different the feature expectations $\mathbb{E}\left(\phi^{\mathrm{m}} \mid R(Z)\right)$ under the various components are. $C_{\mathrm{c}}$ is zero for the generative and fully discriminative estimators, but the pseudolikelihood discriminative estimator pays a penalty for having more than one component.

The misspecification correction $C_{\mathrm{m}}$ is zero when the model is well-specified (in this case, $\phi^{\mathrm{m}}\left|Z \stackrel{d}{=} \phi^{\mathrm{t}}\right| Z$ ), but is in general nonzero under model misspecification. In this latter case, one incurs a nonzero approximation error (defined in (7)) as expected, but we see that there is also a nonzero effect on estimation error.

\subsection{Asymptotics of the Risk}

The following theorem turns Theorem 1 from a statement about the asymptotic distribution of the parameters into one about the risk:
Theorem 2 (Asymptotic distribution of the risk). Let $\Sigma$ be the asymptotic variance as defined in (15). Denote $\dot{\mathcal{R}} \stackrel{\text { def }}{=} \dot{\mathcal{R}}\left(\theta^{\circ}\right)$ and $\ddot{\mathcal{R}} \stackrel{\text { def }}{=} \ddot{\mathcal{R}}\left(\theta^{\circ}\right)$. Then

$$
\sqrt{n}\left(\mathcal{R}(\hat{\theta})-\mathcal{R}\left(\theta^{\circ}\right)\right) \stackrel{d}{\rightarrow} \mathcal{N}\left(0, \dot{\mathcal{R}}^{\top} \Sigma \dot{\mathcal{R}}\right) .
$$

Furthermore, if $\dot{\mathcal{R}}=0$, then

$$
n\left(\mathcal{R}(\hat{\theta})-\mathcal{R}\left(\theta^{\circ}\right)\right) \stackrel{d}{\rightarrow} \frac{1}{2} \operatorname{tr} \mathcal{W}\left(\ddot{\mathcal{R}}^{\frac{1}{2}} \Sigma \ddot{\mathcal{R}}^{\frac{1}{2}}, 1\right),
$$

where $\mathcal{W}(V, n)$ is the Wishart distribution with $n$ degrees of freedom.

Proof. Perform a Taylor expansion of the risk function around $\theta^{\circ}$ :

$$
\begin{aligned}
\mathcal{R}(\hat{\theta})= & \mathcal{R}\left(\theta^{\circ}\right)+\dot{\mathcal{R}}^{\top}\left(\hat{\theta}-\theta^{\circ}\right)+ \\
& \frac{1}{2}\left(\hat{\theta}-\theta^{\circ}\right)^{\top} \ddot{\mathcal{R}}\left(\hat{\theta}-\theta^{\circ}\right)+o\left(\left\|\hat{\theta}-\theta^{\circ}\right\|^{2}\right) .
\end{aligned}
$$

We use a standard argument known as the delta method (van der Vaart, 1998). Multiplying (21) on both sides by $\sqrt{n}$, rearranging terms, and applying Slutsky's theorem, we get (19). However, when $\dot{\mathcal{R}}=0$, the first-order term of the expansion (21) is zero, so we must consider the second-order term to get a non-degenerate distribution. Note that $\ddot{\mathcal{R}}$ is positive semidefinite. Multiplying (21) by $n$ and rearranging yields the following:

$$
n\left(\mathcal{R}(\hat{\theta})-\mathcal{R}\left(\theta^{\circ}\right)\right)=\frac{1}{2} \operatorname{tr}\left(\left[\ddot{\mathcal{R}}^{\frac{1}{2}} \sqrt{n}\left(\hat{\theta}-\theta^{\circ}\right)\right]^{\otimes}\right)+\cdots
$$

Since $\ddot{\mathcal{R}}^{\frac{1}{2}} \sqrt{n}\left(\hat{\theta}-\theta^{\circ}\right) \stackrel{d}{\rightarrow} \mathcal{N}\left(0, \ddot{\mathcal{R}}^{\frac{1}{2}} \Sigma \ddot{\mathcal{R}}^{\frac{1}{2}}\right)$, applying the continuous mapping theorem with the outer product function yields a Wishart as the limiting distribution. Thus, $n\left(\mathcal{R}(\hat{\theta})-\mathcal{R}\left(\theta^{\circ}\right)\right)$ is asymptotically equal in distribution to $\frac{1}{2}$ times the trace of a sample from that Wishart distribution.

We can also understand (20) in the following way. Let $V=\ddot{\mathcal{R}}^{\frac{1}{2}} \Sigma \ddot{\mathcal{R}}^{\frac{1}{2}}$. Note that $\frac{1}{2} \operatorname{tr} \mathcal{W}(V, 1) \stackrel{d}{=}$ $\frac{1}{2} \operatorname{tr}(V \mathcal{W}(I, 1))$, which is the distribution of a weighted sum of independent $\chi_{1}^{2}$ variables, where the weights are determined by the diagonal elements of $V$. The mean of this distribution is $\frac{1}{2} \operatorname{tr}(V)$ and the variance is $\operatorname{tr}(V \bullet V)$, where $\bullet$ denotes elementwise product.

An important question is when we obtain the ordinary $O\left(n^{-\frac{1}{2}}\right)$ convergence (19) versus the much better $O\left(n^{-1}\right)$ convergence (20). A sufficient condition for $O\left(n^{-1}\right)$ convergence is $\dot{\mathcal{R}}\left(\theta^{\circ}\right)=0$. When the model is well-specified, this is true for any consistent estimator.

Even if the model is misspecified, the fully discriminative estimator still achieves the $O\left(n^{-1}\right)$ rate. The 
reason is that whenever a training criterion $m_{\theta}$ is the same (up to constants) as the test criterion $\mathcal{R}(\cdot), \dot{\mathcal{R}}$ vanishes and we obtain the $O\left(n^{-1}\right)$ rate. This is in concordance with a related observation made by Wainwright (2006) that it is better to use the same inference procedure at both training and test time.

When the model is well-specified, there is another appealing property that holds if the training and test criterion are the same up to constants: the asymptotic distribution of the risk depends on only the dimensionality of the exponential family, not the actual structure of the model. In particular, for composite likelihood estimators with one component, $\Sigma=\Gamma^{-1}=$ $\left(-\mathbb{E} \ddot{m}_{\theta^{\circ}}\right)^{-1}=\ddot{\mathcal{R}}^{-1}$. Therefore, $\ddot{\mathcal{R}}^{\frac{1}{2}} \Sigma \ddot{\mathcal{R}}^{\frac{1}{2}}=I_{d}$ and so $n\left(\mathcal{R}(\hat{\theta})-\mathcal{R}\left(\theta^{\circ}\right)\right) \stackrel{d}{\rightarrow} \frac{1}{2} \operatorname{tr} \mathcal{W}\left(I_{d}, 1\right) \stackrel{d}{=} \frac{1}{2} \chi_{d}^{2}$, where $d$ is the number of parameters. This result is essentially another way of looking at the fact that the likelihood ratio test statistic is asymptotically distributed as $\chi^{2}$.

\subsection{Comparing Estimation Errors}

In the previous section, we analyzed the asymptotics of a single estimator. Now, given two estimators, we would like to be able to tell which one is better. In order to compare two estimators, it would be convenient if they converged to the same limit. In this section, we ensure this by assuming that the model is wellspecified and that our estimators are consistent.

Since all parameter estimates are used in the same way for prediction, it suffices to analyze the relative efficiencies of the parameter estimates. The following theorem says that coarser partitionings of $\mathcal{Z}$ generally lead to more efficient estimators:

Theorem 3 (Asymptotic relative efficiency). Let $\hat{\theta}_{1}$ and $\hat{\theta}_{2}$ be two consistent estimators with asymptotic variances $\Sigma_{1}$ and $\Sigma_{2}$ as defined in (15). Assume that $R_{1}$ is constant ( $\hat{\theta}_{1}$ has exactly one component) and $R_{1}(z) \supset R_{2}(z)$ for all $z \in \mathcal{Z}$. If the model is wellspecified, then $\Sigma_{1} \preceq \Sigma_{2}$ ( $\hat{\theta}_{1}$ is no worse than $\hat{\theta}_{2}$ ).

Proof. We first show that $\Gamma_{1}^{-1} \preceq \Gamma_{2}^{-1}$, where $\Gamma_{1}$ and $\Gamma_{2}$ are the sensitivities of the two estimators. Because the model is well-specified, $\Gamma_{k}=\mathbb{E} \operatorname{var}\left(\phi^{\mathrm{t}} \mid R_{k}(Z)\right)$ for $k=1,2$. The assumption $R_{1}(Z) \supset R_{2}(Z)$ means that $R_{2}(Z)$ provides more information about $Z$ than $R_{1}(Z)$; formally, the $\sigma$-fields satisfy $\sigma\left(R_{1}(Z)\right) \subset \sigma\left(R_{2}(Z)\right)$. Thus, we can use Lemma 1 to decompose the variance: $\Gamma_{1}=\mathbb{E} \operatorname{var}\left(\phi^{\mathrm{t}} \mid R_{2}(Z)\right)+\mathbb{E} \operatorname{var}\left[\mathbb{E}\left(\phi^{\mathrm{t}} \mid R_{2}(Z)\right) \mid R_{1}(Z)\right]$. The first term of the right-hand side is exactly $\Gamma_{2}$ and the second term is positive semidefinite, so $\Gamma_{1} \succeq \Gamma_{2}$, which implies $\Gamma_{1}^{-1} \preceq \Gamma_{2}^{-1}$.

Let $C_{\mathrm{c} 1}$ and $C_{\mathrm{c} 2}$ be the component corrections of the two estimators. Note that $C_{\mathrm{c} 1}=0$ because the $R_{1}$ is constant, so $C_{\mathrm{c} 1} \preceq C_{\mathrm{c} 2}$. The misspecification corrections are both zero. Putting these results together yields the theorem.

One might wonder if we really need $R_{1}$ to be constant. Is it not enough to just assume that $R_{1}(z) \supset R_{2}(z)$ (for some coupling of $R_{1}$ and $R_{2}$ )? The answer is no, as the following counterexample shows:

Counterexample Let $\mathcal{Z}=\{1,2,3\}$. The general shape of the distribution is given by the single feature $\phi(1)=1, \phi(2)=3, \phi(3)=2$ and a scalar parameter $\theta$ controls the peakiness of the distribution. Let the true parameter be $\theta^{*}=1$. Consider two estimators: $\hat{\theta}_{1}$ has two components, $r_{1 a}=\{\{1,2\},\{3\}\}$ and $r_{1 b}=\{\{1\},\{2,3\}\} ; \hat{\theta}_{2}$ also has two components, $r_{2 a}=\{\{1,2\},\{3\}\}$ and $r_{2 b}=\{\{1\},\{2\},\{3\}\}$.

Coupling $r_{1 a}$ with $r_{2 a}$ and $r_{1 b}$ with $r_{2 b}$, we have $R_{1}(z) \supset R_{2}(z)$. However, we computed and found that $\Gamma_{1} \approx 4.19$ and $\Gamma_{2} \approx 3.15$, so $\hat{\theta}_{2}$ actually has lower asymptotic variance although it has finer partitionings.

To explain this, note that the contribution of $r_{2 b}$ to the criterion function is zero, so the second estimator is equivalent to just using the single component $r_{2 a}$ $\left(=r_{1 a}\right)$, so the first estimator actually suffers by using the additional component $r_{1 b}$. In general, while we would still expect coarser partitionings to be better even for estimators with many components, this counterexample shows that we must exercise caution.

\subsection{Comparing Estimators}

Finally, we use Theorem 3 to compare the estimation and approximation errors of the generative $\left(\hat{\theta}_{\mathrm{g}}\right)$, fully discriminative $\left(\hat{\theta}_{\mathrm{d}}\right)$, and pseudolikelihood discriminative $\left(\hat{\theta}_{\mathrm{p}}\right)$ estimators. The subscripts $\mathrm{g}, \mathrm{d}, \mathrm{p}$ will be attached to other variables to refer to the quantities associated with the corresponding estimators. In the following corollaries, we use the word "lower" loosely to mean "no more than," although in general we expect the inequality to be strict.

Corollary 1 (Generative versus fully discriminative). (1) If the model is well-specified, $\hat{\theta}_{\mathrm{g}}$ has lower asymptotic estimation error than $\hat{\theta}_{\mathrm{d}}$; both have zero approximation error. (2) If the model is misspecified, $\hat{\theta}_{\mathrm{d}}$ has lower approximation and asymptotic estimation errors than $\hat{\theta}_{\mathrm{g}}$.

Proof. For (1), since $R_{\mathrm{d}}(z) \subset R_{\mathrm{g}}(z)$, we have $\Sigma_{\mathrm{g}} \preceq \Sigma_{\mathrm{d}}$ by Theorem 3. Zero approximation error follows from consistency. For (2), since the discriminative estimator 
achieves the minimum risk in the model family, it has the lowest approximation error. Also, by Theorem 2 and the ensuing discussion, it always converges at a $O\left(n^{-1}\right)$ rate, whereas the generative estimator will in general converge at a $O\left(n^{-\frac{1}{2}}\right)$ rate.

Note that there is a qualitative change of asymptotics in going from the well-specified to the misspecified scenario. This discontinuity demonstrates one weakness of asymptotic analyses: we would expect that for a very minor model misspecification, the generative estimator would still dominate the discriminative estimator for moderate sample sizes, but even a small misspecification is magnified in the asymptotic limit.

In the following toy example where the model is wellspecified, we see concretely that the generative estimator has smaller asymptotic estimation error:

Example Consider a model where $x$ and $y$ are binary variables: $\phi(x, y)^{\top} \theta=\theta_{0} \mathbb{1}[x=0, y=1]+$ $\theta_{1} \mathbb{1}[x=1, y=1]$, where the true parameters are $\theta^{*}=$ $(0,0)$. We can compute $\Gamma_{\mathrm{g}}=\operatorname{var}(\phi)=\frac{1}{16}\left(\begin{array}{cc}3 & -1 \\ -1 & 3\end{array}\right)$ and $\ddot{\mathcal{R}}\left(\theta^{*}\right)=\Gamma_{\mathrm{d}}=\mathbb{E} \operatorname{var}(\phi \mid X)=\frac{1}{16}\left(\begin{array}{cc}2 & 0 \\ 0 & 2\end{array}\right)$. The mean asymptotic estimation error (scaled by $n$ ) of the generative estimator is $\frac{1}{2} \operatorname{tr}\left(\Gamma_{\mathrm{d}} \Gamma_{\mathrm{g}}^{-1}\right)=\frac{3}{4}$ while that of the discriminative estimator is $\frac{1}{2} \operatorname{tr}\left(\Gamma_{\mathrm{d}} \Gamma_{\mathrm{d}}^{-1}\right)=1$.

We now show that fully discriminative estimators are statistically superior to pseudolikelihood discriminative estimators in all regimes, but of course pseudolikelihood is computationally more efficient.

Corollary 2 (Fully discriminative versus pseudolikelihood discriminative). (1) If the model is well-specified, $\hat{\theta}_{\mathrm{d}}$ has lower asymptotic estimation error than $\hat{\theta}_{\mathrm{p}}$; both have zero approximation error. (2) If the model is misspecified, $\hat{\theta}_{\mathrm{d}}$ has lower approximation and asymptotic estimation errors than $\hat{\theta}_{\mathrm{p}}$.

Proof. For (1), since $R_{\mathrm{p}}(z) \subset R_{\mathrm{d}}(z), \Sigma_{\mathrm{d}} \preceq \Sigma_{\mathrm{p}}$ by Theorem 3. Zero approximation error follows from consistency. For (2), the same arguments as the corresponding part of the proof of Corollary 1 apply.

\section{Experiments}

In this section, we validate our theoretical analysis empirically. First, we evaluate the three estimators on a simple graphical model which allows us to plot the real asymptotics of the estimation error (Section 4.1). Then we show that in the non-asymptotic regime, the qualitative predictions of the asymptotic analyses are also valid (Section 4.2).

\subsection{A Simple Graphical Model}

Consider a four-node binary-valued graphical model where $z=\left(x_{1}, x_{2}, y_{1}, y_{2}\right)$. The true model family $p^{*}$ is an Markov random field parametrized by $\theta^{*}=$ $\left(\alpha^{*}, \beta^{*}, \gamma^{*}\right)$ as follows:

$$
\begin{aligned}
\phi(z)^{\top} \theta= & \alpha \mathbb{1}\left[y_{1}=y_{2}\right]+\beta\left(\mathbb{1}\left[x_{1}=y_{1}\right]+\mathbb{1}\left[x_{2}=y_{2}\right]\right)+ \\
& \gamma\left(\mathbb{1}\left[x_{1}=y_{2}\right]+\mathbb{1}\left[x_{2}=y_{1}\right]\right) .
\end{aligned}
$$

To emulate misspecification, we set $\gamma^{*}$ to be nonzero and force $\gamma=0$ during parameter estimation.

In the first experiment, we estimated the variance (by running $10 \mathrm{~K}$ trials) of the estimation error as we increased the number of data points. We set $\alpha^{*}=\beta^{*}=$ 1 for the true model. When $\gamma^{*}=0$ (the model is well-specified), Figures 1(a)-(c) show that scaling the variance by $n$ yields a constant; this implies that all three estimators achieve $O\left(n^{-1}\right)$ convergence.

When the model is misspecified with $\gamma^{*}=0.5$ (Figures $1(\mathrm{~d})-(\mathrm{f})$ ), there is a sharp difference between the rates of the generative and discriminative estimators. The fully discriminative estimator still enjoys the $O\left(n^{-1}\right)$ convergence; scaling by $n$ reveals that the generative and pseudolikelihood discriminative estimators are only attaining a $O\left(n^{-\frac{1}{2}}\right)$ rate as predicted by Theorem 2 (Figure 1(f)). Note that the generative estimator is affected most severely.

Figures $1(\mathrm{~g})-(\mathrm{h})$ demonstrate the non-asymptotic impact of varying the parameters of the graphical model in terms of the total error. In (g), as we increase the amount of misspecification $\gamma$, the error increases for all estimators, but most sharply for the generative estimator. In (h), as we increase the strength of the edge potential $\alpha$, the pseudolikelihood discriminative estimator suffers, the fully discriminative estimator is unaffected, and the generative estimator actually improves.

\subsection{Part-of-speech Tagging}

In this section, we present experiments on part-ofspeech (POS) tagging. In POS tagging, the input is a sequence of words $x=\left(x_{1}, \ldots, x_{\ell}\right)$ and the output is a sequence of POS tags $y=\left(y_{1}, \ldots, y_{\ell}\right)$, e.g., noun, verb, etc. (There are 45 tags total.) We consider the following model, specified by the following features (roughly 2 million total):

$$
\phi(x, y)=\sum_{i=1}^{\ell} \phi_{\text {node }}\left(y_{i}, x_{i}\right)+\sum_{i=1}^{\ell-1} \phi_{\text {edge }}\left(y_{i}, y_{i+1}\right),
$$

where the node features $\phi_{\text {node }}\left(y_{i}, x_{i}\right)$ are a vector of indicator functions of the form $\mathbb{1}\left[y_{i}=a, x_{i}=b\right]$, and 


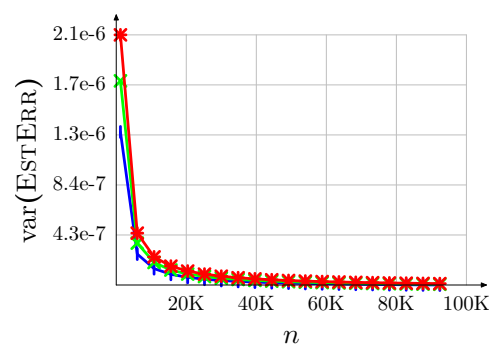

(a) $\alpha^{*}=\beta^{*}=1$

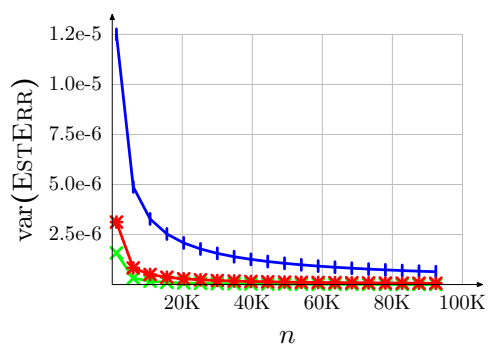

(d) $\alpha^{*}=\beta^{*}=1$

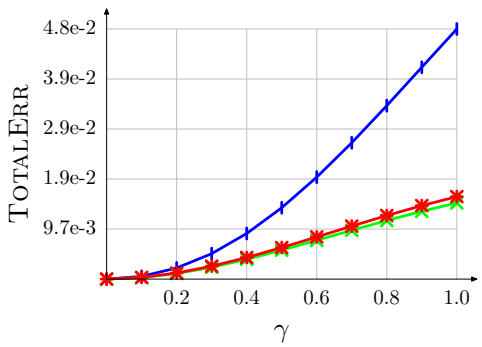

(g) Vary misspecification

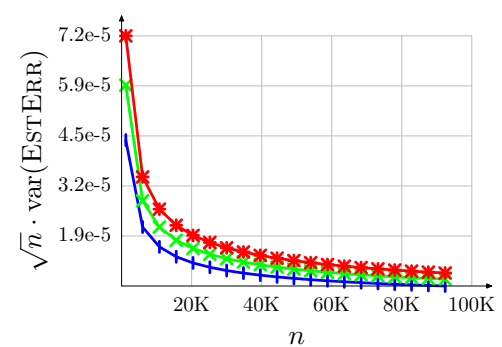

(b) $\alpha^{*}=\beta^{*}=1$

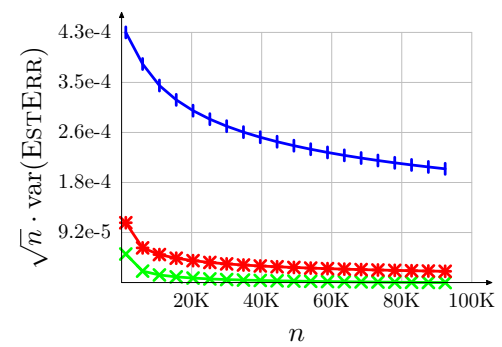

(e) $\alpha^{*}=\beta^{*}=1$

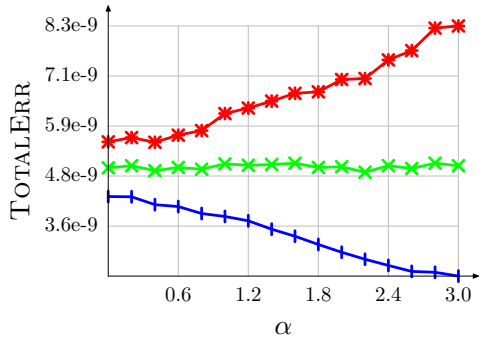

(h) Vary edge potentials

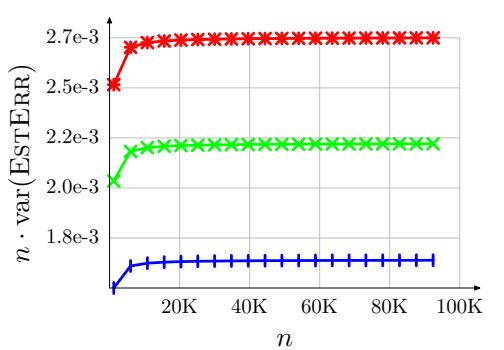

(c) $\alpha^{*}=\beta^{*}=1$

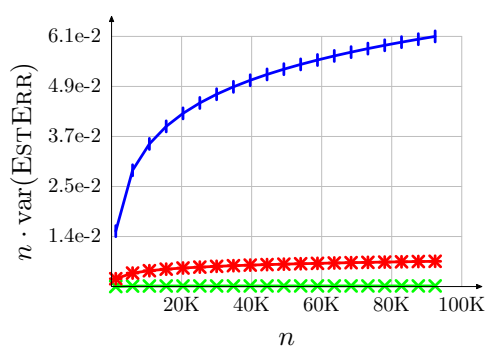

(f) $\alpha^{*}=\beta^{*}=1$

Figure 1. Asymptotics of the simple four-node graphical model. In (a)-(c), $\alpha^{*}=\beta^{*}=1$ and $\gamma^{*}=0$; we plot the asymptotic variance of the estimation error, scaled by $1, \sqrt{n}$, and $n$. In (d)-(f), we repeat with $\gamma^{*}=0.5$. In (g), we take $n=20000$ examples, $\alpha^{*}=\beta^{*}=1$ and vary $\gamma$. In $(\mathrm{h})$, we take $n=20000, \beta^{*}=1, \gamma^{*}=0$ and vary $\alpha$.

the edge features $\phi_{\text {edge }}\left(y_{i}, y_{i+1}\right)$ are a vector of indicator functions of the form $\mathbb{1}\left[y_{i}=a, y_{i+1}=b\right]$. Trained generatively, this model is essentially an HMM, but slightly more expressive. Trained (fully) discriminatively, this model is a CRF.

We used the Wall Street Journal (WSJ) portion of the Penn Treebank, with sections 0-21 for training (38K sentences) and $22-24$ for testing (5.5K sentences). Table 2 (a) shows that the discriminative estimators perform better than the generative one. This is not surprising given that the model is misspecified (language does not come from an HMM).

To verify that the generative estimator is superior when the model is well-specified, we used the learned generative model in the previous experiment to sample 1000 synthetic training and 1000 synthetic test examples. We then applied the estimators as before on this artificial data. Table 2(b) shows that the generative es-

\begin{tabular}{l|cc|cc} 
& \multicolumn{2}{|c}{ Accuracy } & \multicolumn{2}{c}{ Log-loss } \\
\hline & Train & Test & Train & Test \\
\hline Gen. & 0.940 & 0.935 & 4.628 & 4.945 \\
Fully dis. & $\mathbf{0 . 9 7 7}$ & $\mathbf{0 . 9 5 6}$ & $\mathbf{1 . 4 8 0}$ & $\mathbf{3 . 1 2 0}$ \\
Pseudo dis. & 0.975 & 0.955 & 1.562 & 3.170
\end{tabular}

(a) Real data (misspecified)

\begin{tabular}{l|cc|cc} 
& \multicolumn{2}{|c}{ Accuracy } & \multicolumn{2}{c}{ Log-loss } \\
\hline & Train & Test & Train & Test \\
\hline Gen. & 0.989 & $\mathbf{0 . 8 9 8}$ & 0.570 & $\mathbf{7 . 2 9 7}$ \\
Full dis. & $\mathbf{0 . 9 9 2}$ & 0.879 & $\mathbf{0 . 4 0 7}$ & 12.431 \\
Pseudo dis. & 0.990 & 0.891 & 0.469 & 10.840
\end{tabular}

(b) Synthetic data (well-specified)

Table 2. Part-of-speech tagging results. Discriminative estimators outperform the generative estimator (on both test accuracy and log-loss) when the model is misspecified, but the reverse is true when the model is well-specified. 
timator has an advantage over the fully discriminative estimator, and both are better than the pseudolikelihood estimator.

\section{Discussion and Extensions}

We believe our analysis captures the essence of the generative-discriminative distinction: by modeling the input, we reduce the variance of the parameter estimates. In related work, Ng and Jordan (2002) showed that Naive Bayes requires exponentially fewer examples than logistic regression to obtain the same estimation error. The key property needed in their proof was that the Naive Bayes estimator decouples into $d$ independent closed form optimization problems, which does not seem to be the defining property of generative estimation. In particular, this property does not apply to general globally-normalized generative models, but one would still expect those models to have the advantages of being generative.

Given that the generative and discriminative estimators are complementary, one natural question is how to interpolate between the two to get the benefits of both. Our framework naturally suggests two ways to go about this. First, we could vary the coarseness of the partitioning. Generative and discriminative estimators differ only in this coarseness and there is a range of intermediate choices corresponding to conditioning on more or fewer of the input variables. Second, we could take a weighted combination of estimators (e.g., Bouchard and Triggs (2004); McCallum et al. (2006)). For one-parameter models, Lindsay (1988) derived the optimal weighting of the component likelihoods, but unfortunately these results cannot be applied directly in practice.

It would also be interesting to perform a similar asymptotic analysis on other estimators used in practice, for example marginal likelihoods with latent variables, tree-reweighted belief propagation (Wainwright et al., 2003; Wainwright, 2006), piecewise training (Sutton \& McCallum, 2005), etc. Another important extension is to curved exponential families, which account for many of the popular generative models based on directed graphical models.

\section{Conclusion}

We have analyzed the asymptotic distributions of composite likelihood estimators in the exponential family. The idea of considering different partitionings of the outcome space allows a clean and intuitive characterization of the asymptotic variances, which enables us to compare the commonly used generative, discrimina- tive, and pseudolikelihood estimators as special cases. Our work provides new theoretical support for existing intuitions and a basis for developing new estimators which balance the tradeoff between computational and statistical efficiency.

Acknowledgments We thank Peter Bartlett for useful discussions and Simon Lacoste-Julien for comments. We also wish to acknowledge NSF grant 0509559 and a grant from Microsoft Research.

\section{References}

Besag, J. (1975). The analysis of non-lattice data. The Statistician, 24, 179-195.

Bouchard, G., \& Triggs, B. (2004). The trade-off between generative and discriminative classifiers. International Conference on Computational Statistics (pp. 721-728).

Lafferty, J., McCallum, A., \& Pereira, F. (2001). Conditional random fields: Probabilistic models for segmenting and labeling data. International Conference on $\mathrm{Ma}$ chine Learning (ICML).

Lasserre, J. A., Bishop, C. M., \& Minka, T. P. (2006). Principled hybrids of generative and discriminative models. Computer Vision and Pattern Recognition (CVPR) (pp. 87-94).

Liang, P., Klein, D., \& Jordan, M. I. (2008). Agreementbased learning. Advances in Neural Information Processing Systems (NIPS).

Lindsay, B. (1988). Composite likelihood methods. Contemporary Mathematics, 80, 221-239.

McCallum, A., Pal, C., Druck, G., \& Wang, X. (2006). Multi-conditional learning: Generative/discriminative training for clustering and classification. Association for the Advancement of Artificial Intelligence (AAAI).

Ng, A. Y., \& Jordan, M. I. (2002). On discriminative vs. generative classifiers: A comparison of logistic regression and naive Bayes. Advances in Neural Information Processing Systems (NIPS).

Sutton, C., \& McCallum, A. (2005). Piecewise training of undirected models. Uncertainty in Artificial Intelligence (UAI).

van der Vaart, A. W. (1998). Asymptotic Statistics. Cambridge University Press.

Varin, C. (2008). On composite marginal likelihoods. Advances in Statistical Analysis, 92, 1-28.

Wainwright, M. (2006). Estimating the "wrong" graphical model: Benefits in the computation-limited setting. Journal of Machine Learning Research, 7, 1829-1859.

Wainwright, M., Jaakkola, T., \& Willsky, A. (2003). Treereweighted belief propagation algorithms and approximate ML estimation by pseudo-moment matching. Artificial Intelligence and Statistics (AISTATS).

Wainwright, M., \& Jordan, M. I. (2003). Graphical models, exponential families, and variational inference (Technical Report). Department of Statistics, University of California at Berkeley. 